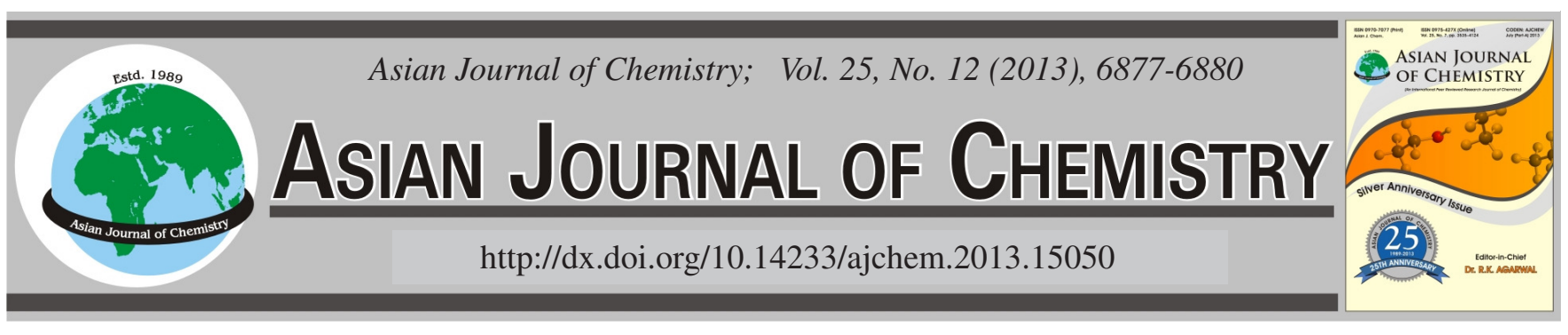

\title{
Electrochemical Fabrication of Graphite Oxide Nanosheets with Stable Photoluminescence and Their Photocatalytic Performance in Visible Light
}

\begin{abstract}
X.M. ZHAO ${ }^{1, *}$ and M.J. MALIK ${ }^{2}$
${ }^{1}$ College of Chemistry, Chemical Engineering and Material Science, Soochow University, Renai Road, 199, Suzhou 215123, Jiangsu, P.R. China ${ }^{2}$ Department of Chemical Engineering, University of Waterloo, Waterloo N2L 3G1, Ontario, Canada

*Corresponding author: Tel: +86 18703562876; E-mail: zhaoxiaomei9@126.com

(Received: 27 December 2012;

Accepted: 5 June 2013)

AJC-13592

Graphite oxide nanosheets surface-passivated by ethylene glycol (GONs-EG) were synthesized by a green electrochemical method.
GONs-EG exhibited blue luminescence and high up-conversion photoluminescence, also showed peroxidase mimetic function and visible-
light-sensitive photocatalytic activity.
Key Words: Graphite oxide, Electrochemical method, Photoluminescence, Photocatalytic.
\end{abstract}

\section{INTRODUCTION}

Fluorescent nanoparticles have been a hot topic of current interest due to their promising applications covering electrooptics to bionanotechnology. Meanwhile, obtaining different fluorescent nanoparticles with high water solubility and biocompatibility is urgent in order to decrease the negative influence on biological system and to enhance their biological labeling efficiency. Recently, the emergence of photoluminescent carbon quantum dots or carbon nanomaterials has received much attention and presented exciting opportunities for searching benign fluorescent nanomaterials ${ }^{1,2}$, as they may gradually replace traditional semiconductor quantum dots due to their superiority in chemical inertness, biocompatibility and low toxicity ${ }^{3}$.

Carbon nanomaterials are usually prepared by laser ablation of graphite ${ }^{4}$, electrochemical oxidation of graphite ${ }^{5}$, vapour deposition of $\operatorname{soot}^{6}$, microwave synthesis ${ }^{7}$ and so forth. In addition, these carbon nanomaterials are usually need further surface-passivated by polymers such as poly(ethylene glycol) $(\mathrm{PEG})^{5}$, ionic liquid ${ }^{8}$ and liquid crystalline polymers ${ }^{9}$ for their further applications. Surface-passivation of carbon nanomaterials can not only lead to a enhanced photoluminescent intensity but also exhibit a series of superiorities such as tunable chemical activity, biocompatibility and low toxicity, which notably increased the potential applications in bioimaging, disease detection and drug delivery. No matter what the methods are used for synthesis and passivation of carbon nanomaterials as mentioned above, all suffered from the drawbacks of complex synthesis or needing specified experimental set up, expensive preparation and separation procedures. Therefore, there is a huge challenge to search an easy and green method to synthesize and passivate carbon nanomaterials. Especially, due to the nontoxic property, high water solubility and biocompatibility of ethylene glycol, surface modification of carbon nanomaterials by ethylene glycol to improve the biocompatibility for bio-application has been a hot topic research ${ }^{10}$.

Herein, we present a new easy and green electrochemical method to prepare graphite oxide nanosheets (GONs) surfacepassivated by ethylene glycol (EG). They exhibit blue luminescence and upconverted emission in water solution, implying the GONs-EG are very stable and have potential applications in biological labeling. Interestingly, GONs-EG also possess excellent peroxidase mimetic function and photocatalytic ability for methyl orange degradation, indicating the potential of GONs-EG as a medium for water splitting and pollutant degradation under solar illumination.

\section{EXPERIMENTAL}

All chemicals were used as received without further purification. Two high-purity graphite rods $(99.99 \%)$, placed parallel with a separation of $7.5 \mathrm{~cm}$ and inserted as electrodes into a mixture solution of $400 \mathrm{~mL}$ ultra-pure water (UPW), $100 \mathrm{~mL} \mathrm{H} \mathrm{O}_{2}, 100 \mathrm{~mL}$ ethylene glycol and $1 \mathrm{~g} \mathrm{NH}_{4} \mathrm{H}_{2} \mathrm{PO}_{4}$. Detailed experiment set up is shown in Fig. 1a. Static potential of $30 \mathrm{~V}$ was applied to the two electrodes. After $72 \mathrm{~h}$ under stirring, the anode graphite rod was corroded and then a deepyellow solution gradually appeared in the reactor. The solution was taken out of the reactor and successively filtered with slow 
speed quantitative filter paper. The resultant solution was centrifugated at $10000 \mathrm{rpm}$ for $0.5 \mathrm{~h}$ in order to remove small graphite. Then the obtained solution was transferred to a dialysis bag (MWCO: 14000) to remove the redundant $\mathrm{NH}_{4} \mathrm{H}_{2} \mathrm{PO}_{4}, \mathrm{H}_{2} \mathrm{O}_{2}$ and ethylene glycol, thereby leaving behind water soluble GONs-EG in the solution.

Photocatalytic activities test: A sample ( $3 \mathrm{~mL})$ containing 10 ppm of methyl orange, 10 ppm GONs-EG and $\mathrm{H}_{2} \mathrm{O}_{2}(3 \%)$ was placed in a quartz colorimetric ware. The $\mathrm{pH}$ value of the solution was adjusted to 7 by buffer solution. The reaction was held at $298 \mathrm{~K}$ and stirring. There were two compared experiments: one was in the dark area and the other was exposed to visible light $(150 \mathrm{~W}$, Xenon lamp). The methyl orange in this reaction was quantified by the measurement of the decay of absorbance at $\lambda_{\max }=465 \mathrm{~nm}$ at a certain of time.

Detection method: Transmission electron micrograph (TEM) was taken on a FEI-Tecnai F20. Fourier transform infrared (FT-IR) was recorded on a Ventrix (Brukle) spectrophotometer. X-ray photoelectron spectroscopy (XPS) was carried out by using a KRATOS Axis ultra-DLD X-ray photoelectron spectrometer. Fluorescent spectrophotometry was recorded on FluoroMax 4 (Horiba Jobin Yvon) spectrophotometer.

\section{RESULTS AND DISCUSSION}

A simple and detailed experiment set up was used for synthesizing large-scale GONs-EG (Fig. 1a). The TEM image (Fig. 1b) shows that GONs-EG mostly consist of a few layers of graphene and their diameters are mainly distributed in the range of $30-50 \mathrm{~nm}$, suggesting the uniform size distribution of as-prepared GONs-EG. HRTEM image inserted in Fig. $1 \mathrm{~b}$ further illuminates the essence of the as-synthesized GONs-EG composed of a few layers of graphene oxide. FTIR spectrum (Fig. 1c) of GONs-EG showed the peaks located at 3442.19 (hydroxyl OH), 2884, 1394, 883 (aromatic Ar-H, $\mathrm{C}-\mathrm{H}$ ), 1644 (aromatic C=O), 1081, 1036 (epoxide/ether C-OC) and $1468 \mathrm{~cm}^{-1}$ (carboxy $\mathrm{C}=\mathrm{C}$ ), which indicates a successful oxidation and the surface-passivated by ethylene glycol. The XPS spectrum (inset of Fig. 1c) of $\mathrm{C} 1 \mathrm{~s}$ exhibits a mass of oxidation groups $(\mathrm{C}-\mathrm{O}, \mathrm{C}=\mathrm{O})$ in GONs-EG. Furthermore, the zeta potential of aqueous GONs-EG dispersion was measured to be $-42.1 \mathrm{mV}$, confirming the electrostatic stabilization of GONs-EG dispersion.

On the basis of the above results, we speculate the formation of GONs-EG due to the electrochemical oxidation and esterification, as shown in the detailed scheme in Fig. 1a (right). At first, through the electrochemical oxidation, the $s p^{2}$-hybridized $(\mathrm{C}=\mathrm{C})$ carbon atoms in aromatic rings of graphite were partially decomposed to functional oxygen groups such as $\mathrm{COOH}, \mathrm{COH}, \mathrm{C}=\mathrm{O}$. Secondly, due to the existence of plenty of $\mathrm{OH}$ groups on ethylene glycol and based on the esterification of GONs and EG, as a result, GONs-EG were formed. Meanwhile, $\mathrm{H}_{2} \mathrm{O}_{2}$ and $\mathrm{NH}_{4} \mathrm{H}_{2} \mathrm{PO}_{4}$ can accelerate the graphite oxidation and electrons transferring rate during the electrochemical process. At last, GONs-EG was exfoliated and a GONs-EG solution was formed. Photographs of the GONs-EG aqueous solution have been taken under visible light (left) and $365 \mathrm{~nm}$ UV light (right), from which we can see that a high blue

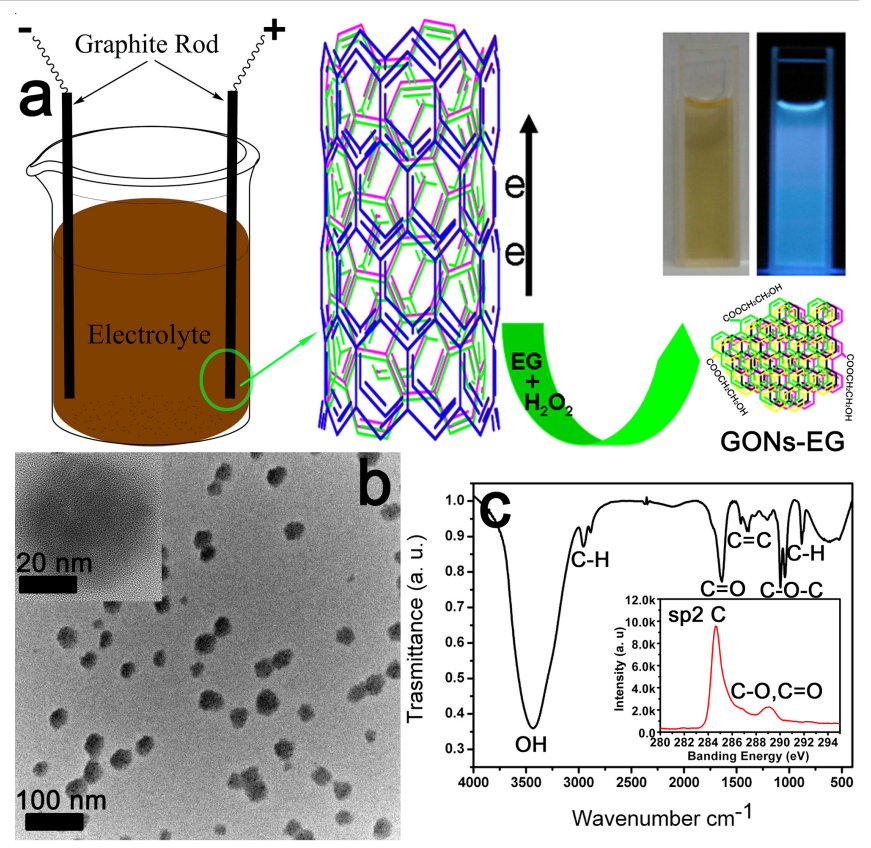

Fig. 1. (a) Illustration of experimental set up and detailed scheme for the preparation of GONs-EG; (b) TEM and HRTEM images of GONsEG; (c) FT-IR and XPS of C1s (inset) spectra of GONs-EG

photoluminescent was observed under the $365 \mathrm{~nm}$ UV light. The blue photoluminescent should be contributed to the presence of isolated $s p^{2}$ clusters within the carbon-oxygen $s p^{3}$ matrix, which leads to the localization of e-h pairs, thus facilitating radiative recombination of small clusters. The calculation carried out by Goki Eda et al. suggested that $s p^{2}$ clusters of several conjugated repeating units yielded band gaps consistent with blue emission ${ }^{11}$.

To further explore the optical properties of as-synthesized GONs-EG, a detailed photoluminescent study was carried out by using light at different wavelengths (200, 250, 300, 350, 400, 450, 500, 550, 600, 650 and $700 \mathrm{~nm}$ ) as excitation. Fig. 2a shows the photoluminescent spectra of GONs-EG. It is obvious that all of the samples exhibit the visible emissions covering blue-to-red wavelength range, located at 512, 426, 420, 429, 475, 513, 546, 582, 621, 424, $431 \mathrm{~nm}$, respectively. A closed observation shows that for some excitations there is second emission peak and the photoluminescent emission can be extended into NIR wavelength range. The photoluminescent in such carbon systems is a consequence of geminate recombination of localized e-h pairs in $s p^{2}$ clusters, which essentially behave as the luminescence centers or chromophores ${ }^{12}$.

The up-converted photoluminescent materials can convert a longer wavelength excitation (e.g., NIR light) to shorter wavelength fluorescence (e.g., visible light), which possess prominent potentials in biological and clinical applications and photocatalyst design for applications in environmental and energy issues. Most of the up-converted fluorescence materials reported were inorganic crystals doped with rare-earth elements ${ }^{13}$ or encapsulating organic dyes or quantum dots in the silica shell ${ }^{14}$. Until now, there are few materials which can display both visible emission and up-conversion photoluminescent. Here, the as-synthesized GONs-EG can be excited by NIR wavelength light and emit bright photoluminescent in visible 
spectral band. Fig. $2 \mathrm{~b}$ shows the photoluminescent spectra of GONs-EG excited by 750, 800, 850, 900, 950, $1000 \mathrm{~nm}$ laser with the up-converted emissions located at about 458, 473, 495, 510 (414), 523 (414), 543(414) nm, respectively (the number in bracket ( ) is the second up-converted peak). Remarkably, an interesting phenomenon appeared when the excitation light wavelength increased $50 \mathrm{~nm}$ successively. The shifting about $1.05 \mathrm{eV}$ between the energy of upconverted emission light (Em) and excitation light (Ex) was unchanged. The linear relationship between emission light and excitation light and the function of the fit line was $\mathrm{Em}=1.00 \mathrm{Ex}+\mathrm{dE}$ with $\mathrm{dE}=$ $1.05 \mathrm{eV}$. Since, there is a constant energy $(1.05 \mathrm{eV})$ difference between the excitation and emission light, ASPL mechanism can illuminate this upconverted luminescence process ${ }^{15,16}$. When a bunch of low-energy photons excite the electrons of the $p$ orbital, the $p$ electrons would transit to a high-energy state such as the LUMO and then the electrons transit back to a lowenergy state. Thus, an upconverted photoluminescent is emitted when the electrons transit back to the s orbital ${ }^{8}$. The photoluminescent quantum yields of the GONs-EG measured using rhodamine $\mathrm{B}$ as a reference is high to $2.78 \%$ which was comparable with those of reported luminescent carbon nanoparticles.
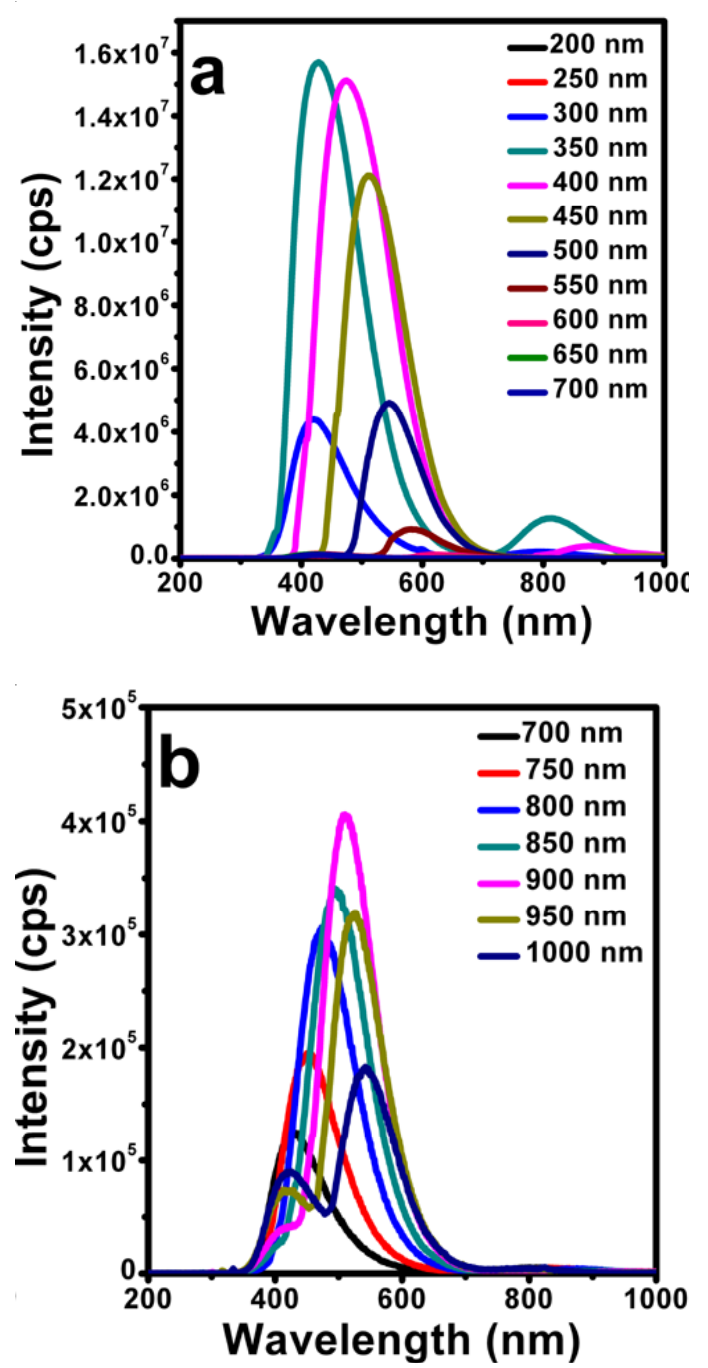

Fig. 2. Photoluminescent spectra of the GONs-EG at different excitation wavelengths: (a) $200-700 \mathrm{~nm}$, step is $50 \mathrm{~nm}$. (b) $700-1000 \mathrm{~nm}$, step is $50 \mathrm{~nm}$
Furthermore, we highlighted the peroxidase mimetic behaviour and photocatalytic activity of the as-prepared GONsEG. In an exploratory experiment to demonstrate the intrinsic enzyme mimetic activity of GONs-EG, degradation of methyl orange was examined in the presence of $\mathrm{H}_{2} \mathrm{O}_{2}$. Fig. 3a revealed no degradation efficiency when $\mathrm{H}_{2} \mathrm{O}_{2}$ was mixed with methyl orange. While GONs-EG was present, a significant degradation (160 $\mathrm{min}, 82.2 \%$ ) of the methyl orange was observed, indicating the peroxidase mimetic function of GONs-EG. This effect could be explained by the formation of an activated transition complex $\left[\mathrm{C} \cdots \mathrm{H}_{2} \mathrm{O}_{2}\right]$, which transfers electron density from the $\pi$-system of the GONs-EG to the peroxide molecule during the decomposition of $\mathrm{H}_{2} \mathrm{O}_{2}$ process ${ }^{17}$. Other interesting result was observed, when this reaction system was exposed to visible light, a better catalytic activity of GONs-EG were found (Fig. 3b), in which methyl orange can be photodegrade completely within $80 \mathrm{~min}$. This phenomenon may be attributed to the apparent band gap (2.4-4.3 eV) of the GONs-EG, it is capable of absorbing visible lightand generated a large amount of photoreactive species, such as $\mathrm{O}^{2-}$ and $\cdot \mathrm{OH}$, with very strong oxidation capability, which account
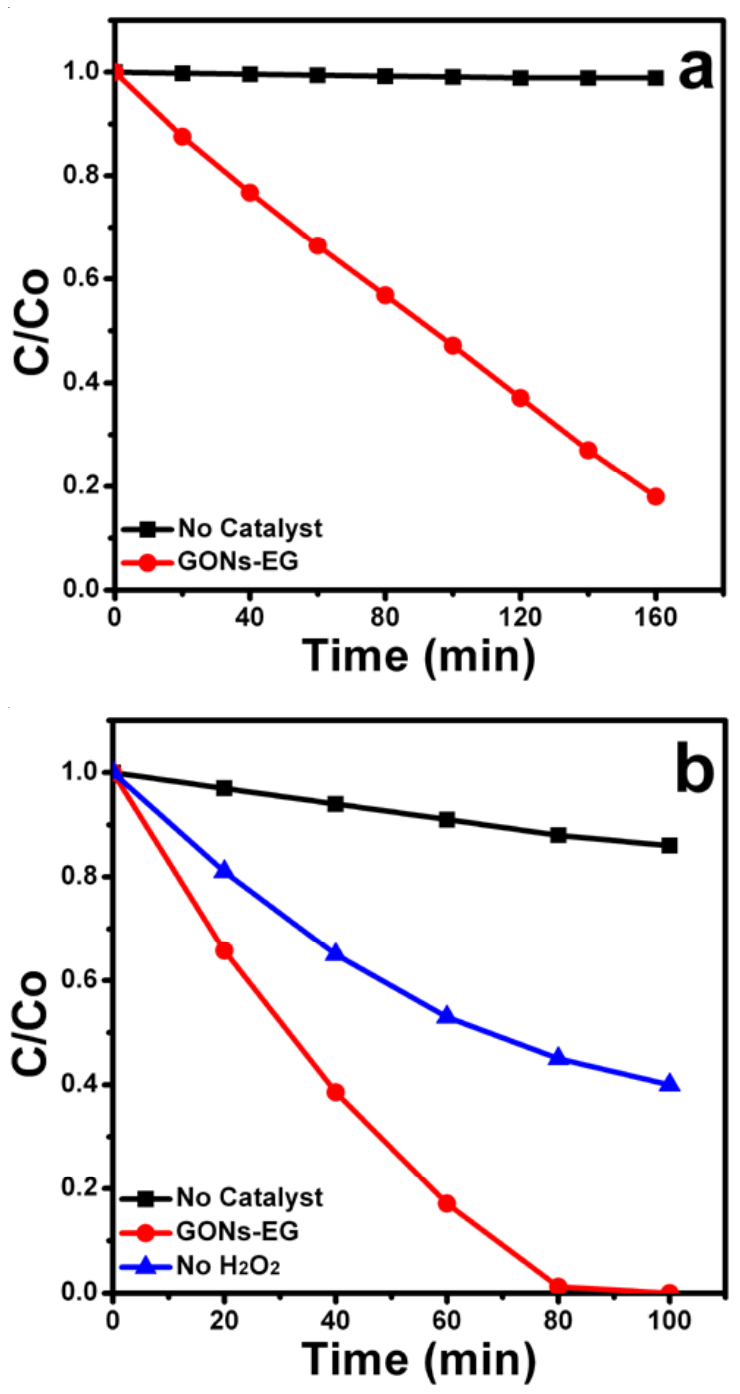

Fig. 3. (a) Degradation of methyl orange, (b) Photodegradation of methyl orange in the presence of $\mathrm{H}_{2} \mathrm{O}_{2}$. Blue line (tagged with $>$ ): Photodegrade methyl orange without $\mathrm{H}_{2} \mathrm{O}_{2}$ under visible light. 
for the higher photocatalytic activity of the GONs-EG ${ }^{18,19}$. In addition, for GONs, the conduction band is the antibonding $\pi^{*}$ orbital $^{20}$. The efficient methyl orange photodegradation of GONs-EG can also be attributed to the fact that GONs-EG can be well dispersed in water and the carbon atoms on the sheets are therefore accessible to protons, so that $\mathrm{H}_{2} \mathrm{O}$ and $\mathrm{H}_{2} \mathrm{O}_{2}$ can readily transform to ${ }^{\circ} \mathrm{OH}$ which possess high oxidation ability during photoreaction. These active species can recover oxidation ability after catalytic reaction by accepting photogenerated electrons from the carbon atoms. This phenomenon was also illustrated by blue line (Fig. 3b, tagged with -). When there is no $\mathrm{H}_{2} \mathrm{O}_{2}$ in reaction system, GONs-EG can also photodegrade methyl orange under visible light, which indicated that GONs-EG is visible light sensitive.

\section{Conclusion}

In summary, GONs-EG with blue luminescence and upconverted emission were obtained by a green electrochemical method. Furthermore, it was demonstrated that GONs-EG have excellent peroxidase mimetic function and visible-light-driven photocatalystic ability, indicating the potential of GONs-EG as a medium for water splitting and pollutant degradation under solar illumination.

\section{ACKNOWLEDGEMENTS}

Finance support from Soochow University are gratefully acknowledged.

\section{REFERENCES}

1. Y.B. Zhang, Y.W. Tan, H.L. Stormer and P. Kim, Nature, 438, 201 (2005).

2. J.S. Bunch, A.M. Zande, S.S. Verbridge, I.W. Frank, D.M. Tanenbaum, J.M. Parpia, H.G. Craighead and P.L. McEuen, Science, 315, 490 (2007).
3. L. Cao, X. Wang, M.J. Meziani, F.S. Lu, H.F. Wang, P.G. Luo, Y. Lin, B.A. Harruff, L.M. Veca, D. Murray, S.Y. Xie and Y.P. Sun, J. Am. Chem. Soc., 129, 11318 (2007).

4. Y.P. Sun, B. Zhou, Y. Lin, W. Wang, K.A.S. Fernando, P. Pathak, M.J. Meziani, B.A. Harruff, X. Wang, H.F. Wang, P.G. Luo, H. Yang, M.E. Kose, B.L. Chen, L.M. Veca and S.Y. Xie, J. Am. Chem. Soc., 128, 7756 (2006).

5. L.Y. Zheng, Y.W. Chi, Y.Q. Dong, J.P. Lin and B.B. Wang, J. Am. Chem. Soc., 131, 4564 (2009).

6. H.P. Liu, T. Ye and C.D. Mao, Angew. Chem. Int. Ed., 46, 6473 (2007).

7. H. Zhu, X.L. Wang, Y.L. Li, Z.J. Wang, F. Yang and X.R. Yang, Chem. Commun., 5118 (2009).

8. J.H. Shen, Y.H. Zhu, C. Chen, X.L. Yang and C.Z. Li, Chem. Commun., 2580 (2011).

9. N.G. Sahoo, H.K.F. Cheng, L. Li, S.H. Chan, Z. Judeh and J.H. Zhao, Adv. Funct. Mater, 19, 3962 (2009).

10. K. Yang, S. Zhang, G.X. Zhang, X.M. Sun, S.T. Lee and Z. Liu, Nano Lett., 10, 3318 (2010).

11. G. Eda, Y.Y. Lin, C. Mattevi, H. Yamaguchi, H.A. Chen, I.S. Chen, C.W. Chen and M. Chhowalla, Adv. Mater, 22, 505 (2010).

12. T. Heitz, C. Godet, J.G. Boureé, B. Drevillon and J.P. Conde, Phys. Rev. B, 60, 6045 (1999)

13. F. Zhang, R.C. Haushalter, R.W. Haushalter, Y.F. Shi, Y.C. Zhang, K.L. Ding, D.Y. Zhao and G.D. Stucky, Small, 7, 1972 (2011).

14. J.A. Fan, C.H. Wu, K. Bao, J.M. Bao, R.Z. Bardhan, N.J. Halas, V.N. Manoharan, P. Nordlander, G. Shvets and F. Capasso, Science, 328, 1135 (2010).

15. J.R. Santos, M.I. Vasilevskiy and S.A. Filonovich, Phys. Rev. B, 78, 245422 (2008).

16. X.Y. Wang, W.W. Yu, J.Y. Zhang, J. Aldana, X.G. Peng and M. Xiao, Phys. Rev. B, 68, 125318 (2003).

17. V.F. Lapko, I.P. Gerasimyuk, V.S. Kuts and Y. Tarasenko, Russ. J. Phys. Chem. A, 84, 934 (2010).

18. T.F. Yeh, J.M. Syu, C. Cheng, T.H. Chang and H.S. Teng, Adv. Funct. Mater, 20, 2255 (2010).

19. Y. Li, Y. Hu, Y. Zhao, G.Q. Shi, L.E. Deng, Y.B. Hou and L.T. Qu, Adv. Mater., 23, 776 (2011).

20. J. Ito, J. Nakamura and A. Natori, J. Appl. Phys., 103, 113712 (2008). 